\title{
Performance of the 16-Item Informant Questionnaire on Cognitive Decline for the Elderly (IQCODE) in an Arabic-Speaking Older Population
}

\author{
Thien Kieu Thi Phung ${ }^{a} \quad$ Monique Chaaya $^{b} \quad$ Khalil Asmar $^{b} \quad$ Samir Atweh ${ }^{c}$ \\ Husam Ghusn ${ }^{d}$ Rose Mary Khoury ${ }^{b}$ Martin Prince ${ }^{e}$ Gunhild Waldemar ${ }^{a}$ \\ a Danish Dementia Research Center, Department of Neurology, Neuroscience Center, \\ Rigshospitalet, University of Copenhagen, Copenhagen, Denmark; bepartment of \\ Epidemiology and Population Health, Faculty of Health Sciences, and Departments of \\ ${ }^{\mathrm{c}}$ Neurology and d Geriatrics, American University of Beirut Medical Center, Beirut, Lebanon; \\ e Department of Health Service and Population Research, Institute of Psychiatry, \\ King's College London, London, UK
}

\section{Key Words}

Dementia $\cdot$ Illiteracy $\cdot$ Education $\cdot$ Informant-based screening $\cdot$ Arabic

\begin{abstract}
Background/Aim: The North African and Middle Eastern region has high illiteracy rates among older people, making direct cognitive testing challenging. Validated screening instruments for dementia in Arabic are lacking. We aimed to validate the Arabic version of the 16item Informant Questionnaire on Cognitive Decline for the Elderly (A-IQCODE 16) for screening for dementia through an informant. Methods: 236 Lebanese participants older than 65 years, 143 with normal cognition and 93 with mild-to-moderate dementia according to the DSM-IV criteria, and their informants were recruited. Half of the participants had no formal education. Interviewers blinded to the cognitive status of the participants administered the A-IQCODE 16 to the informants. The ability of the A-IQCODE 16 to screen for dementia was evaluated against the DSM-IV diagnoses. Results: The A-IQCODE 16 had excellent overall predictive power (area under the receiver operator characteristic curve $=0.96$ ). A cutoff point of $>3.34$ yielded the best sensitivity (92.5\%) and specificity (94.4\%) for dementia screening. At this cutoff point, the discriminatory ability of the A-IQCODE 16 was comparable between participants with and those without formal education. Conclusion: The A-IQCODE 16 is not biased by education and is therefore useful as a brief screening tool for dementia among Arabic-speaking older adults with low education.

(C) 2015 S. Karger AG, Basel
\end{abstract}




\section{Introduction}

As its population is rapidly aging at an unprecedented rate [1], North Africa and the Middle Eastern region [also called by the World Health Organization the 'Eastern Mediterranean Region' (EMR)] ranks second in the world regarding the rate at which the number of people with dementia is expected to increase, i.e. 1.2 million people in 2010 rising to 2.6 million in 2030 and 6.2 million in 2050, an increase of $125 \%$ by 2030 and $438 \%$ by 2050 [2]. Therefore, timely diagnosis and interventions for people with dementia as well as psychosocial support for their caregivers are becoming increasingly important in this region.

An accurate diagnosis is required prior to any intervention, and cognitive assessment is key to the diagnostic process for dementia. The high rate of illiteracy among older people in the EMR renders cognitive assessment very challenging, since commonly used cognitive screening tests, such as the Mini-Mental State Examination (MMSE) or the Montreal Cognitive Assessment (MoCA) [3, 4], require reading, writing, and arithmetic skills. It has been shown that low education and illiteracy have a significant impact on most neuropsychological tests across a wide range of cognitive domains, making it difficult to interpret the test results [5]. The great challenge of direct cognitive testing of illiterate older people underlines the importance of indirect cognitive assessment through interviews with a knowledgeable informant.

The Informant Questionnaire on Cognitive Decline for the Elderly (IQCODE) is a brief screening assessment for dementia based on an interview with an informant, usually a family caregiver [6]. It has been well validated in different languages, cultures, and populations with low education, showing psychometric properties comparable to those of the commonly used MMSE [7]. As a screening tool for dementia in community and clinical settings, the IQCODE correlates well with several commonly used cognitive tests $[8,9]$. It has a high reliability and is a good measure of general cognitive decline [10-12]. It is not affected by premorbid intelligence or education and therefore useful as a complementary screening tool for dementia among older people with limited education $[12,13]$.

A short form of the IQCODE, containing 16 items, was subsequently developed, which highly correlated with the original version and possessed comparable validity [14]. The aims of this study were therefore to evaluate the ability of the Arabic version of the 16-item IQCODE (A-IQCODE 16) to screen for dementia in an Arabic-speaking study population with a high prevalence of illiteracy. It was part of a validation study of three cognitive assessments in Arabic using the same study population: (a) the 10/66 Dementia Research Group diagnostic assessment [15], (b) the Rowland Universal Dementia Assessment Scale (RUDAS) [16], and (c) the A-IQCODE 16.

\section{Methods}

\section{Instrument}

The IQCODE was developed in 1989 and comprises 26 questions [6]. From the original version, the short 16-item IQCODE version was developed in 1994 [14]. Its performance is essentially identical to that of the original version [14]. The validity of the short IQCODE as a screening instrument for dementia was subsequently confirmed in other studies, with sensitivity ranging from 79 to $100 \%$ and specificity from 68 to $100 \%$ $[13,17,18]$. The IQCODE requires an informant who has known the older individual in question for at least 10 years to respond to the questions. This 10 -year time frame was conveniently chosen based on the rationale that the survival period after dementia onset usually does not exceed 10 years [19]. However, other studies found that using a 5-year time frame did not affect the validity of the IQCODE [20,21]. In our study, the informants had to have observed the cognitive status of the study population over a period of at least 1 year, since establishing cognitive decline over 1 year is sufficient to diagnose dementia. The International Classification of Diseases 10th revision (ICD-10) criteria for dementia only require the observation of cognitive decline 
Phung et al.: Performance of the 16-Item Informant Questionnaire on Cognitive

Decline for the Elderly (IQCODE) in an Arabic-Speaking Older Population

over a minimum period of 6 months [22]. For the short IQCODE to be valid, a maximum of 2 items are allowed to be missing [19].

The informant rates the changes in cognitive functioning of the participant over the time period of 1 year or more. Each question of the IQCODE is rated on a 5-point scale from 1 ('much improved') to 5 ('much worse'), with 3 representing 'no change'. The sum of scores is averaged over the 16 items to give a score of 1-5. Higher scores indicate greater impairment. The cutoff scores for cognitive impairment defined in previous studies ranged from 3.38 to $3.53[13,14,17,18]$.

\section{Translation}

The A-IQOCDE 16 was translated from English into Arabic without any need for modification. Subsequently, it was independently back-translated into English and compared with the original English version to verify the quality of the translation. Then, meetings were held with elderly community representatives, physicians, interviewers (research workers), and epidemiologists to discuss the conceptual validity of the A-IQCODE 16 in Lebanese cultural and linguistic contexts. The translated A-IQOCDE 16 was then pilot tested under field conditions on 10 individuals in order to assess the relevance and acceptability of the questions. A sample size of 10 was sufficient to assess whether the questions were acceptable, understandable, and answerable to the informants. The individuals were persons older than 65 years who frequented the social organizations for the elderly and volunteered to take part in the pilot test. They were assessed by physicians from the research team, who clinically diagnosed 1 participant with mild dementia (DSM-IV criteria). The A-IQCODE 16 was well tolerated and answered by the informants of these individuals. There was no need for further modification.

The Arabic version of the 10/66 DRG Diagnostic Instrument for Dementia is available upon request either to T.K.T. Phung at thien.phung@mail.mcgill.ca or M. Chaaya at mchaaya @aub.edu.lb. It will also be made available on the IQCODE website at http://crahw.anu.edu.au/risk-assessment-tools/informantquestionnaire-cognitive-decline-elderly (accessed June 6, 2015).

Training

The interviewers, 3 neurology fellows, 1 geriatrics fellow, 1 family medicine resident, and 1 non-medical university graduate (Master of Public Health), were trained in administering the A-IQCODE 16.

\section{Participants}

The study population was recruited from social organizations for the elderly, hospital-based neurological and geriatric clinics, and community-based primary care clinics. The inclusion criteria were: (1) age 65 years and older; (2) having normal cognition (controls) or mild-to-moderate dementia (cases), and (3) having a knowledgeable informant who is able to give an independent assessment of the elderly participant's health and cognitive status. The exclusion criteria were: (1) a severe somatic (such as severe chronic obstructive pulmonary disease, heart failure, or end-stage renal failure) or psychiatric illness (such as severe depression or psychosis), since the diagnosis of dementia could not have been ascertained without an extensive medical workup and neuropsychological testing, and (2) clinically suspected mild cognitive impairment (MCI) according to the core clinical criteria for MCI by the National Institute on Aging-Alzheimer's Association workgroups [23]. Dementia diagnoses for the study population were established by clinicians according to the DSM-IV [24]. Dementia severity was rated using the Clinical Dementia Rating (CDR) scale [25]. Mental status was assessed with the Geriatric Mental State (GMS) [26], and depression was diagnosed using GMS/AGECAT stage 1 output, which has a sensitivity of $90 \%$ for diagnosing depression among older people with and without dementia in developing countries [27].

Subjects with dementia were recruited via two ways: (1) by prior service contact (neurologists and geriatricians referred the cases they diagnosed as MCI and dementia) and (2) by snowball sampling (community health workers from primary care clinics and lay staff from social organizations identified individuals with cognitive problems).

Following a predefined protocol, independent physicians confirmed the dementia diagnoses according to the DSM-IV criteria and rated dementia severity according to the CDR (Appendix 1) [24, 25]. For the cases identified by prior service contact, the independent physicians performed a neurological examination and reviewed the case notes to complete a standard assessment which included any history of dementia illness, mental state examination, cognitive tests, physical examination including vital signs, and relevant paraclinical investigations (such as complete blood count, electrolytes, serum glucose, renal function tests, thyroid function tests, liver function tests, CT scans of the brain, and any other relevant measures). If the clinical 
information from the case notes was insufficient, the independent physicians from the research team, in consensus with the referring specialists from the clinics, carried out bedside cognitive assessments (MMSE) of the study population and interviewed the study population and their informants, inquiring into problems with cognitive, social, and occupational function, any history of cognitive symptoms (onset and course), and any family history. The independent physicians diagnosed dementia according to the DSM-IV diagnostic criteria for dementia based on the synthesis of all the clinical information from the patients and the informants through their clinical assessments.

For the cases identified through snowball sampling, the independent physicians (neurology fellows) carried out the same standard assessments according to the protocol described above. They performed neurological examinations, carried out cognitive testing (MMSE), and interviewed the study population and their informants, but they could not carry out any paraclinical investigations due to the lack of resources. These patients resided in the community.

The criteria that separated MCI and dementia are those defined by the National Institute on AgingAlzheimer's Association workgroups [23]: (1) there is evidence of concern about a change in cognition; (2) impairment in one or more cognitive domains (such as memory, executive function, attention, language, and visuospatial skills) that is greater than would be expected from the patient's age and educational background; (3) preservation of independence of functional abilities in daily life, requiring minimal aids or assistance, and (4) not demented (the cognitive changes should be sufficiently mild that there is no evidence of a significant impairment in social or occupational functioning).

Validated cognitive tests in Arabic were lacking at the time of the study. The MMSE and the MoCA have been translated into Arabic and validated $[28,29]$. As expected, the MMSE was shown to be educationally biased [29], but it is still the most commonly used cognitive test in the clinical setting and in research. The MoCA has not been validated in Arabic among illiterate older individuals [28]. At the time of our study, the MoCA was being validated in the Lebanese population. Since the MMSE cannot adequately assess cognitive deficits in MCI, trained neuropsychologists were not available, and we did not have the financial resources to carry out supplementary assessments such as biomarker testing and neuroimaging, we had to exclude patients with clinically suspected MCI from the study.

The control group was nominated by the participating social organizations and clinics, e.g. the physicians at the clinics and lay staff from the social organizations nominated individuals they believed to have normal cognition to be controls. There was a risk of misclassification, meaning that some very mild cases of dementia could be misclassified as normal cognition. With regard to the neurological and geriatric clinics, the risk was negligible, since the controls were identified by specialists who had been seeing the individuals as their patients over a long period of time for noncognitive conditions. As for the community-based social organizations, the risk of misclassification should have been lower than the population prevalence of dementia estimated for the EMR $(<6 \%)[2]$.

The informants were identified as the persons who were most involved in the care of the older individuals in the study population or most knowledgeable about the mental and health status of the latter through regular contacts over a time period of at least 1 year. The informants could therefore be lay persons (relatives, friends, or social workers) or health professionals (nurses or physiotherapists). For the older participants living in the community, the informants were mainly family caregivers, and in the nursing homes, it was mainly the nurses in charge of the older participants who were the informants. The interviewers were blinded to the cognitive status of the participants while administering the A-IQOCDE 16 to the informants. Data collection was completed between March 2012 and February 2013.

\section{Ethics}

The Institutional Review Board of the American University of Beirut, Lebanon, approved the study. All participants gave written informed consent, or their relatives gave consent on behalf of the older individuals with impaired decision making capacity. In subjects with dementia, decision making capacity was assessed through a checklist to ensure they completely comprehended the nature of the study and their rights were protected. Immediately after the consent procedure, a post-consent quiz containing 17 questions assessing comprehension (yes/no and short answers; maximum possible score $=17$ ) was read aloud to the study population by the interviewer. The questions inquired about the illness being studied, the procedure, potential risks and benefits, voluntariness, the possibility of withdrawal, confidentiality, and who to contact for further questions or complaints. The correct answers were scored in the scoring sheet provided (Appendix 2). In case of wrong answers, educational procedures (repetition and more detailed explanations of items that the participants had difficulty understanding) were carried out by the interviewer, and the number of questions 
Phung et al.: Performance of the 16-Item Informant Questionnaire on Cognitive

answered correctly on subsequent trials would be added to the previous scores. The participant had a maximum of 3 attempts. The total score had to be 17 (100\% correct). This procedure has been used by Dunn et al. [30] and Wirshing et al. [31]. Informed consent and decision making capacity assessments were carried out by an independent research worker.

\section{Statistical Analysis}

The overall predictive ability of the A-IQCODE 16 was evaluated as the area under the receiver operating characteristic curve (AUROC; sensitivity plotted against 1 - specificity). The AUROC was used to choose the cutoff point with the best sensitivity and specificity for the A-IQCODE 16. At the chosen cutoff point, the following parameters were estimated for the A-IQCODE 16 against a clinician's dementia diagnosis: sensitivity, specificity, positive predictive value, negative predictive value, and Youden's index [(sensitivity + specificity) - 1], which summarized sensitivity and specificity in a single measure at the chosen cut points. A sensitivity analysis stratified by type of residence (community vs. nursing home) and informant (lay person vs. health professional) was carried out to assess the performance of the A-IQCODE 16 in different settings. Pearson's $\chi^{2}$ test was used to compare proportions, together with Fisher's two-tailed exact test when there were cells with a frequency $<5$. Student's t test and one-way ANOVA were used to compare means between groups to determine whether gender, education (no formal vs. formal education), type of informant (lay person vs. health professional), type of relationship (spouse, child, other relative, friend/social worker, and health professional), and the presence of depression affect the A-IQCODE 16 score. The 'no formal education' group included people who were illiterate or could barely read and write. The 'formal education' group included those with primary education and above. Spearman's correlation was used to determine if there was any association between age and A-IQCODE 16 score. The mean A-IQCODE 16 score was reported with standard deviation $( \pm$ SD). An $\alpha$ level of $5 \%$ was used to indicate statistical significance. Data were analyzed using SPSS 19.

\section{Results}

The social organizations and clinics referred 318 individuals older than 65 years; 37 subjects were excluded according to the predefined criteria, 8 could not be contacted, and 29 refused to participate (fig. 1). The response rate was $88.4 \%$. Thus, 244 older individuals were recruited and their informants identified. Those who refused to participate or did not respond were mainly from the hospital-based private neurology clinics and therefore most likely belonged to a socioeconomically advantaged group. In all, 241 subjects or $98.8 \%$ of the study population were recruited from the social organizations, community-based primary care clinics, and hospital-based geriatric clinics, which are charity organizations, providing free food, medicine, and social and medical services for older people. These places of recruitment reflect a diversity of socioeconomic statuses, mainly from low to medium.

All 16 questions were answered for 229 individuals (93.9\%) of the study population. Five (2\%) missed 1 answer, $2(0.8 \%)$ missed 2 answers, and 8 (3.3\%) missed $>2$ answers (range 3-8 answers). Of the 8 subjects who were excluded from the analysis because their A-IQCODE 16 had $>2$ answers missing (fig. 1), 7 individuals had dementia, 7 resided in nursing homes, and 5 had lay informants. The questions that the informants most commonly failed to answer were about 'learning how to operate a new gadget/machine in the house' (3.3\%), 'learning new things in general' $(2.8 \%)$, 'handling financial matters' $(2.8 \%)$, and 'handling everyday arithmetic problems' (3.2\%). The informants found the questions inapplicable as the older individuals in question were usually not asked to undertake these tasks during their daily life.

Of the 236 individuals of the study population whose data were analyzed, $50.0 \%$ had no formal education (were illiterate or could barely read and write); $19.1 \%$ had completed primary education (grades 1-5), 13.2\% intermediate education (grades 6-9), 10.6\% secondary school (grades 10-12), and 6.8\% university. The informants for the subjects of the study population living in the community were mainly relatives (spouses: $24.9 \%$; children: 
Phung et al.: Performance of the 16-Item Informant Questionnaire on Cognitive

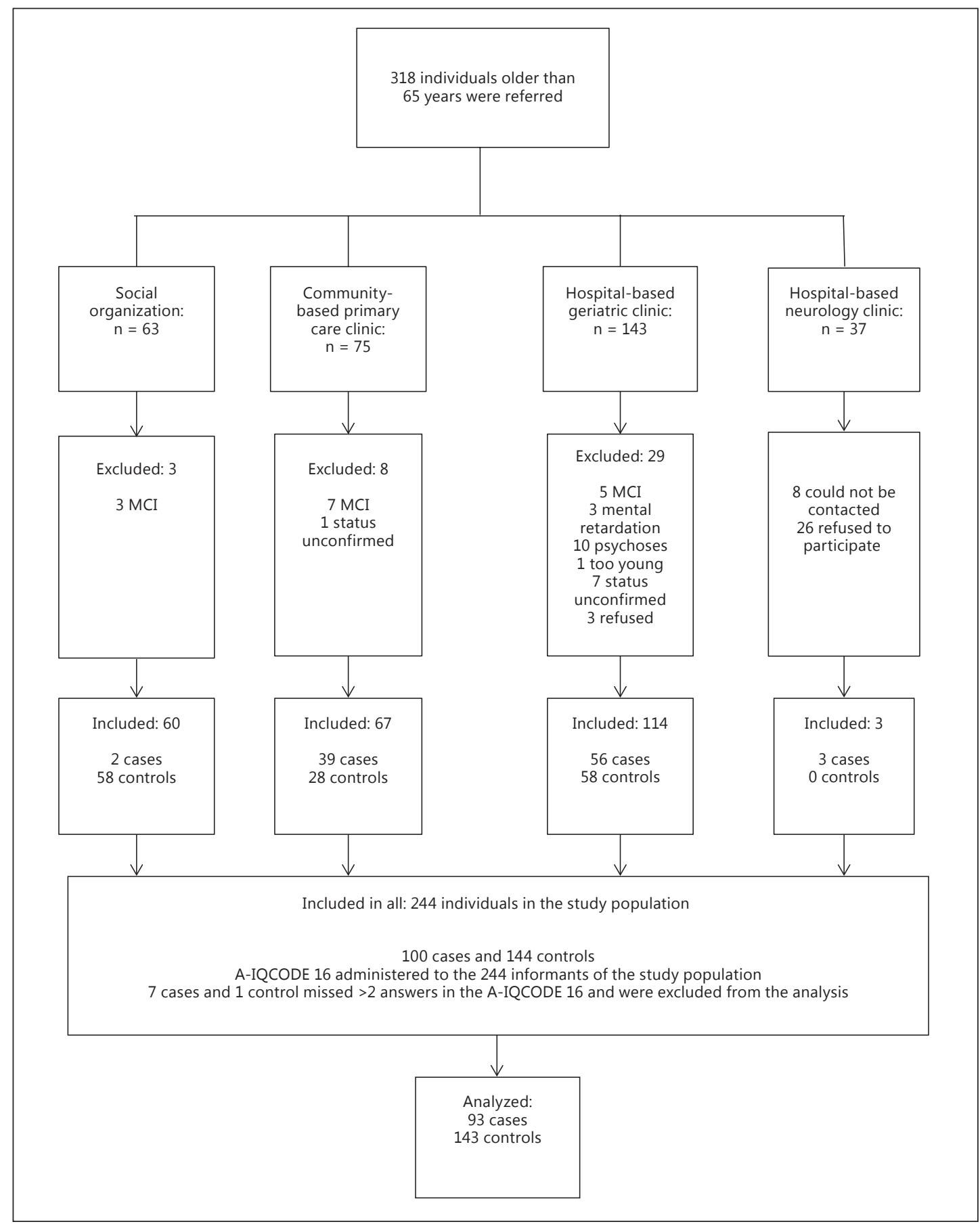

Fig. 1. Recruitment flowchart. Status unconfirmed: severe depression, brain lesions, or severe somatic illnesses. Psychoses: bipolar disorder, schizophrenia.

49.7\%; other relatives: 8.5\%; friends/neighbors: $7.2 \%$; social workers: $8.5 \%$; nurses: $1.3 \%$ ). The informants in the nursing homes were mainly health professionals (nurses and physiotherapists: $77.1 \%$; social workers: $7.2 \%$; children: $8.4 \%$; other relatives: $6.0 \%$; friends: $1.2 \%$ ). There was no statistically significant difference in the proportions of lay (relatives, friends, social workers) and health professional informants (nurses, physiotherapists) between cases 
Phung et al.: Performance of the 16-Item Informant Questionnaire on Cognitive Decline for the Elderly (IQCODE) in an Arabic-Speaking Older Population

Table 1. Sociodemographic characteristics of the study population $(n=236)$

\begin{tabular}{|c|c|c|c|}
\hline \multirow[t]{2}{*}{ Variables } & \multicolumn{2}{|c|}{ Cognitive status } & \multirow[t]{2}{*}{$\mathrm{p}$ value } \\
\hline & dementia & normal cognition & \\
\hline Total & $93(39.4)$ & $143(60.6)$ & \\
\hline Gender & & & 0.301 \\
\hline Male & $29(31.2)$ & $54(37.8)$ & \\
\hline Female & $64(68.8)$ & $89(62.2)$ & \\
\hline Age, years & $81.8 \pm 7.7$ & $77.0 \pm 7.9$ & $<0.001$ \\
\hline Education & & & 0.011 \\
\hline No formal education (illiterate, read and write) & $56(60.2)$ & $62(43.4)$ & \\
\hline Formal education (primary education and above) & $37(39.8)$ & $81(56.6)$ & \\
\hline Informant & & & 0.236 \\
\hline Lay person (relative, friend, social worker) & $63(67.7)$ & $107(74.8)$ & \\
\hline Health professional (nurse, physiotherapist) & $30(32.3)$ & $36(25.2)$ & \\
\hline Residence & & & 0.004 \\
\hline Community & $50(53.8)$ & $103(72.0)$ & \\
\hline Nursing home & $43(46.2)$ & $40(28.0)$ & \\
\hline Depression & & & 0.894 \\
\hline Present & $47(50.5)$ & 71 (49.7) & \\
\hline Absent & $46(49.5)$ & $72(50.3)$ & \\
\hline
\end{tabular}

Values are presented as $\mathrm{n}(\%)$ or the mean \pm SD.

and controls (table 1). Gender was equally distributed among cases and controls. Half of the study population had depression according to AGECAT stage 1 output; the proportions of individuals in the study population with depression were equally distributed among cases and controls. The cases were significantly older than the controls, and a significantly higher proportion of cases had no formal education. Most controls resided in the community (table 1). Fifty individuals of the study population had mild dementia, and 43 had moderate dementia. There was no statistically significant difference between the distribution of mild and moderate dementia among cases recruited from the community and the distribution among cases recruited from the nursing homes $(p=0.193)$.

The A-IQCODE 16 items showed a high level of internal consistency, with Cronbach's $\alpha$ of 0.97. A principle component factor analysis showed that one strong general factor accounted for $72 \%$ of the common variance. Since homogeneity of variances was violated (Levene's test of homogeneity of variance, $p<0.005$ ), Welch ANOVA and the Games-Howell post hoc test were used to test for differences in mean A-IQCODE 16 scores between participants with normal cognition, those with mild dementia, and those with moderate dementia. The mean A-IQCODE 16 scores were statistically significantly different between these three groups $(\mathrm{p}<0.0005)$. The mean A-IQCODE 16 score increased from the normal cognition group (3.06 $\pm 0.31)$ to the mild dementia group $(3.84 \pm 0.50)$ and the moderate dementia group $(4.49 \pm$ 0.51 ), in that order. The Games-Howell post hoc analysis revealed that the increase in mean A-IQCODE 16 score from normal cognition to mild dementia $(0.78$; 95\% CI: 0.60-0.96) was statistically significant $(\mathrm{p}<0.0005)$, as was the increase from mild dementia to moderate dementia (0.64; 95\% CI: 0.39-0.89, $\mathrm{p}=<0.0005)$. Using the subset of controls, Student's test did not show any statistically significant differences in mean A-IQCODE 16 scores between men and women $(p=0.863)$, between those with no formal education and those with formal education $(\mathrm{p}=0.202)$, and between lay informants and health professional informants $(\mathrm{p}=$ 0.734). There was a trend that the mean scores of controls with depression were slightly 
Dementia

and Geriatric

Cognitive Disorders

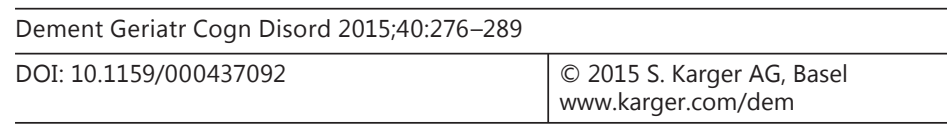

Phung et al:: Performance of the 16-Item Informant Questionnaire on Cognitive Decline for the Elderly (IQCODE) in an Arabic-Speaking Older Population

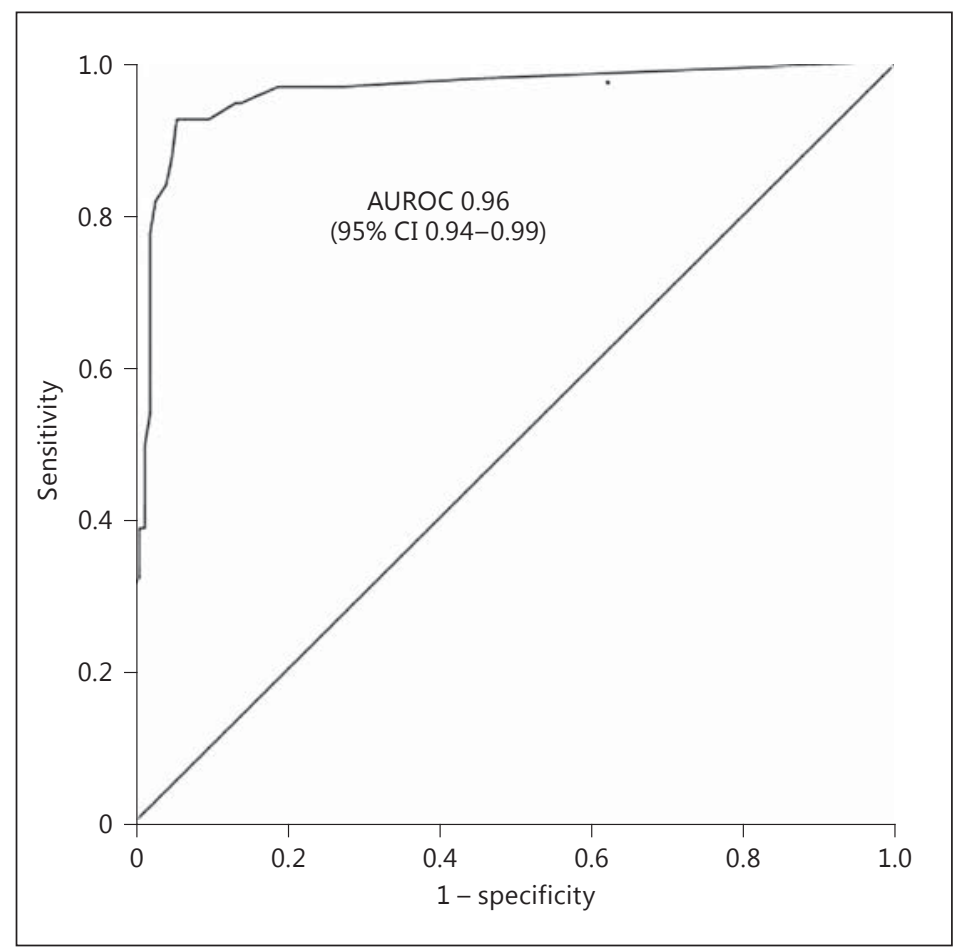

Fig. 2. AUROC.

higher than the mean scores of those without depression (depressed: $3.11 \pm 0.20$; not depressed: $3.02 \pm 0.40 ; p=0.07)$. There was no significant correlation between age and A-IQCODE 16 score $\left(r_{s}=0.109, p=0.193\right)$. A separate one-way ANOVA for cases and controls showed no significant association between type of relationship (spouse, child, other relative, friend/social worker, and health professional) and mean A-IQCODE 16 score either for controls ( $p=0.222$ ) or cases ( $p=0.905)$. Tukey's post hoc comparisons showed no significant difference in A-IQCODE 16 mean scores in pairwise comparison across the five types of relationship.

A-IQCODE 16 showed an excellent overall predictive power regarding dementia, with an AUROC of 0.96 (95\% CI: 0.94-0.99; fig. 2). The performance of the A-IQCODE 16 at different cutoff points is presented in table 2 . The cutoff point with the best balance between sensitivity and specificity was $>3.34$.

At the cutoff point of $>3.34$, a sensitivity analysis stratified by type of informant lay person vs. health professional) showed that the A-IQCODE 16 had excellent overall predictive power regarding dementia when administered to both types of informant, as illustrated by the AUROC (table 3). At the chosen cutoff point of $>3.34$, which was still the cutoff point with the best balance of sensitivity and specificity for both groups of informants, there was no significant difference in sensitivity, specificity, positive predictive value, and false positive rate for people with depression when the A-IQCODE 16 was administered to these two different types of informant. The false positive rate among people with depression calculated the proportion of individuals in the study population who had normal cognition and who were depressed and were incorrectly screened as having dementia by the A-IQCODE 16 at the cutoff point of $>3.34$. Similarly, the sensitivity analysis stratified by type of residence showed that the discriminatory ability of the A-IQCODE 16 in these two types of setting was comparable. Furthermore, education did not significantly affect the psychometric properties of the A-IQCODE 16 at this cutoff point (table 3). 
Table 2. Discriminatory ability of the A-IQCODE 16 at different cutoff points $(n=236)$

\begin{tabular}{lllllll}
\hline $\begin{array}{l}\text { Cutoff point } \\
\text { (dementia if A-IQCODE } \\
16 \text { score was ...) }\end{array}$ & Sensitivity & Specificity & $\begin{array}{l}\text { Youden's } \\
\text { index }\end{array}$ & PPV & NPV & $\begin{array}{l}\text { Correctly } \\
\text { identified }\end{array}$ \\
\hline$>3.22$ & $94.6(87.3-98.0)$ & $86.7(79.8-91.6)$ & 0.813 & $82.2(73.4-88.7)$ & $96.1(90.7-98.6)$ & $89.8(85.3-93.1)$ \\
$>3.28$ & $92.5(84.6-96.7)$ & $90.2(83.8-94.3)$ & 0.827 & $86.0(77.3-91.9)$ & $94.9(89.3-97.7)$ & $91.1(86.8-94.1)$ \\
$>3.34$ & $92.5(84.6-96.7)$ & $94.4(88.9-97.4)$ & 0.869 & $91.5(83.4-96.0)$ & $95.1(89.7-97.8)$ & $93.6(89.8-96.1)$ \\
$>3.40$ & $87.1(78.2-92.9)$ & $95.1(89.8-97.8)$ & 0.822 & $92.0(83.8-96.5)$ & $91.9(86.0-95.6)$ & $91.9(87.8-94.8)$ \\
$>3.47$ & $83.9(74.5-90.4)$ & $95.8(90.7-98.3)$ & 0.797 & $92.9(84.5-97.1)$ & $90.1(84.0-94.2)$ & $91.1(86.8-94.1)$ \\
\hline
\end{tabular}

All values except Youden's index are percentages with 95\% CI in parentheses. PPV = Positive predictive value; NPV = negative predictive value.

Table 3. Performance of the A-IQCODE 16 stratified by type of informant, residence, and education at the cutoff point of $>3.34$ (dementia if A-IQCODE 16 score >3.34)

\begin{tabular}{|c|c|c|c|c|c|}
\hline & AUROC & Sensitivity & Specificity & PPV & FPR depression \\
\hline \multicolumn{6}{|l|}{ Informant } \\
\hline Lay person & $\begin{array}{l}0.944(0.904,0.984) \\
n=170\end{array}$ & $\begin{array}{l}88.9(77.8,95.0) \\
n=170\end{array}$ & $\begin{array}{l}93.5(86.5,97.1) \\
n=170\end{array}$ & $\begin{array}{l}88.9(77.8,95.0) \\
n=170\end{array}$ & $\begin{array}{l}11.6(2.9,20.2) \\
n=52\end{array}$ \\
\hline Health professional & $\begin{array}{l}0.995(0.984,1.000) \\
n=66\end{array}$ & $\begin{array}{l}100.0(85.9,100.0) \\
n=66\end{array}$ & $\begin{array}{l}97.2(83.8,99.9) \\
n=66\end{array}$ & $\begin{array}{l}96.8(81.5,99.8) \\
n=66\end{array}$ & $\begin{array}{l}5.3(-4.8,15.4) \\
n=19\end{array}$ \\
\hline \multicolumn{6}{|l|}{ Residence } \\
\hline Community & $\begin{array}{l}0.950(0.910,0.989) \\
n=153\end{array}$ & $\begin{array}{l}90.0(77.4,96.3) \\
n=153\end{array}$ & $\begin{array}{l}93.2(86.0,97.0) \\
n=153\end{array}$ & $\begin{array}{l}86.5(73.6,94.0) \\
n=153\end{array}$ & $\begin{array}{l}11.6(2.9,20.2) \\
n=52\end{array}$ \\
\hline Nursing home & $\begin{array}{l}0.980(0.950,1.000) \\
n=83\end{array}$ & $\begin{array}{l}95.3(82.9,99.2) \\
n=83\end{array}$ & $\begin{array}{l}97.5(85.3,99.9) \\
n=83\end{array}$ & $\begin{array}{l}97.6(85.9,99.9) \\
n=83\end{array}$ & $\begin{array}{l}5.3(-4.8,15.4) \\
n=19\end{array}$ \\
\hline \multicolumn{6}{|l|}{ Education } \\
\hline No formal education & $\begin{array}{l}0.950(0.904,0.995) \\
n=118\end{array}$ & $\begin{array}{l}96.4(86.6,99.4) \\
n=118\end{array}$ & $\begin{array}{l}93.5(83.5,97.9) \\
n=118\end{array}$ & $\begin{array}{l}93.1(82.5,97.8) \\
n=118\end{array}$ & $\begin{array}{l}10.5(4.2,24.1) \\
n=38\end{array}$ \\
\hline Formal education & $\begin{array}{l}0.908(0.838,0.987) \\
n=118\end{array}$ & $\begin{array}{l}86.5(70.4,94.9) \\
n=118\end{array}$ & $\begin{array}{l}95.1(87.2,98.4) \\
n=118\end{array}$ & $\begin{array}{l}88.9(73.0,96.4) \\
n=118\end{array}$ & $\begin{array}{l}9.1(3.1,23.6) \\
n=33\end{array}$ \\
\hline
\end{tabular}

All values except AUROC are in percentages with 95\% CI in parentheses. PPV = Positive predictive value; FPR = false positive rate among those in the study population with depression and normal cognition.

\section{Discussion}

In this study population of Arabic-speaking older participants, half of whom had no formal education, the A-IQCODE 16 demonstrated excellent psychometric properties to screen for dementia in the community as well as in nursing homes. The strength of this study was the standardized translation process, training and supervision of interviewers, and case ascertainment. Clinicians from teaching hospitals in Lebanon established dementia diagnosis and rated dementia severity using well-validated clinical criteria.

The study also has some weaknesses. Due to resource constraints, in-depth neuropsychological assessments and paraclinical investigations to investigate clinically suspected MCI cases and cases with severe somatic and psychiatric disorders were not available. This could have led to a spectrum bias. Severe somatic and psychiatric cases were mostly found in the 
nursing homes, whereas MCI cases mostly had their residence in the community. This, together with the inclusion of participants from a long-term care setting (where the prevalence of dementia was higher and the informants were mostly health professionals) and the use of highly trained physicians as interviewers, could have led to an overestimation of the screening ability of the A-IQCODE 16. On the other hand, the snowball sampling approach used in the social organizations and community-based primary care clinics could have led to a misclassification of people with mild dementia as controls. The staff at these organizations could have missed cognitive symptoms in some people and, thus, did not nominate them for clinical assessment. This nondifferential misclassification could have led to an underestimation of the screening ability of the A-IQCODE 16. However, the problem of overestimation clearly outweighed the issue of underestimation in this study. Since the interviewers in the study were mainly physicians - as well as one university graduate (Master of Public Health) - who underwent rigorous training and were closely supervised throughout their fieldwork, equally excellent results may not be found under less optimal conditions. Due to the same resource constraints, we could not determine any subtypes. The etiologies were diverse, most likely following the same breakdown of subtypes in clinical or community samples, with Alzheimer's disease being the most common, followed by vascular dementia and mixed Alzheimer's disease/vascular dementia.

Consistent with results from previous studies, the A-IQCODE 16 possessed a high level of internal consistency and measured a single general factor of cognitive decline [10-12]. It could effectively discriminate between normal cognition, mild dementia, and moderate dementia. Its scores were not affected by gender, age, or education. The cutoff point with the best balance of sensitivity and specificity was $>3.34$, even when the sensitivity analysis was stratified by type of informant (lay person vs. health professional). The sensitivity analysis stratified by type of informant and residence showed that the A-IQCODE 16 demonstrated very good psychometric properties when administered to both lay informants and health professionals, and when used in the community or in an institution. It is therefore a valid screening test for dementia in the community and in institutions (table 3).

Previous studies showed that depression was positively correlated with IQCODE score, probably because the informants had trouble distinguishing anxiety and depression from cognitive decline $[10,14]$. The presence of depression tended to increase the A-IQCODE 16 score $(p=0.07)$ in our study, though not statistically significantly.

The type of relationship (spouse, child, other relative, friend/social worker, or health professional) did not affect the A-IQCODE 16 mean scores among cases and controls in this study. The same finding was reported in another study [12]. Previous studies have demonstrated positive correlations between A-IQCODE 16 score and informant characteristics such as informant anxiety, depression, and poor quality of the relationship with the older participant $[8,17]$, but our study was not designed to address this question.

As mentioned in the Introduction, we also validated the RUDAS, a brief cognitive test to screen for dementia, in the same patient population. We demonstrated that it possessed good psychometric properties (sensitivity: 83\%; specificity: 85\%) to screen for dementia among older individuals with low education [16]. Although the RUDAS was designed to minimize the effect of culture and education, the items testing praxis and visuoconstruction (drawing a cube) proved to be very difficult for illiterate older people with normal cognition. The consensus today is that patient- and informant-based data should be combined to enhance screening and diagnostic accuracy. In Australia, the IQCODE has been combined with the MMSE to improve screening accuracy [32]. In Switzerland, a very brief instrument called 'BrainCheck' to optimize case finding for general practitioners has recently been developed, using a shorter version of the IQCODE (7 items) combined with patients' subjective impairment and cognitive testing (clock drawing test) [33]. Following the same rationale, when screening 
for dementia among older people in developing countries where the prevalence of illiteracy is high, the IQCODE and the RUDAS can be combined in order to provide complementary information on cognitive status, to minimize the education bias of the RUDAS, and to reduce the potential effects of patients' and informants' characteristics such as depression on the IQCODE.

\section{Conclusions}

This study has demonstrated the good discriminatory ability of the A-IQCODE 16 as a brief screening tool for dementia among Arabic-speaking older adults, many of whom had no formal education. It requires little training, is brief, and is very easy to administer. The questionnaire has good acceptability and applicability among Arabic-speaking elderly people. It can be used to screen for dementia in the community as well as in nursing homes in combination with a cognitive test such as the RUDAS and is recommended when the target population is illiterate or has a low level of education.

\section{Appendix 1: Independent Clinical Assessment (Developed by the 10/66 DRG)}

January-July 2012

Name of clinician:

A. Patient information

Name:

Age:

Date of birth (if known):

Address:

Telephone number:

B. Caregiver/informant information

Name:

Relationship with participant:

Address:

Telephone number:

C. Patient interview

1. Presenting complaints

2. History

3. Mental state examination

- Appearance and behavior

- Mood

- Cognitive status

4. Physical examination

5. Relevant investigations

D. Informant interview

1. Any problems with cognitive/social/occupational functioning?

2. History of onset of disorder ('What were the first symptoms? When were they first noticed?')

3. History of course of disorder ('Which symptoms appeared early on, which later? Was the progression gradual or stepwise? If stepwise, were sudden deteriorations associated with clear-cut cerebrovascular events?')

4. Family history of dementia or other significant condition?

E. Summary 
Phung et al.: Performance of the 16-Item Informant Questionnaire on Cognitive

Decline for the Elderly (IQCODE) in an Arabic-Speaking Older Population

\section{Appendix 2: Post-Consent Quiz Validation Study}

Post-Consent Quiz Validation Study

Name

Date of Interview

Name of Interviewer

In case of inadequate scoring after each attempt, educational procedures (repetition and more detailed explanations of items that the participants have difficulty in understanding) will be carried out by the interviewer to achieve the desired score. The participant has a maximum of three attempts. The total score has to be $100 \%$ correct.

\begin{tabular}{|c|c|c|}
\hline Post-consent quiz & Correct answer & Score \\
\hline What is the illness being studied? & Dementia & \\
\hline What is the purpose of the study? & $\begin{array}{l}\text { To make sure a new cognitive test can diagnose } \\
\text { dementia accurately }\end{array}$ & \\
\hline What is required of you as a participant? & $\begin{array}{l}\text { To be interviewed by a doctor and to be } \\
\text { examined briefly by a doctor }\end{array}$ & \\
\hline What is required of your caregiver (relative or friend) & To be interviewed by a doctor & \\
\hline $\begin{array}{l}\text { What is the total time required for the } \\
\text { interview? }\end{array}$ & Approximately $1 \mathrm{~h}$ & \\
\hline $\begin{array}{l}\text { Does participation involve any physical or } \\
\text { emotional risk beyond the risks of daily life? }\end{array}$ & No & \\
\hline What is the benefit of the study? & New test to diagnose dementia & \\
\hline Do you receive any direct benefit? & No & \\
\hline Is participation voluntary? & Yes & \\
\hline If you want to, when can you quit the study? & Any time & \\
\hline Do you need a reason to quit the study? & No & \\
\hline $\begin{array}{l}\text { Will quitting the study affect your relationship } \\
\text { to the American University of Beirut in any way? }\end{array}$ & No & \\
\hline $\begin{array}{l}\text { Will quitting the study affect your future } \\
\text { medical care in any way? }\end{array}$ & No & \\
\hline
\end{tabular}

Can your personal information be linked to the result No, publications and presentation of data will be in any way? anonymous

How can confidentiality be secured?

Person-identifiable data will be kept safe in a secured place and destroyed after the required retention period (5 years)

Where can you find the number to call if you have any In the informed consent form question?

Where can you find the number to call if you have any In the informed consent form complaints about your right as a participant?

\section{Total scores}

First attempt

Second attempt

Third attempt

Score 1 for each correct answer (total 17). 


\begin{tabular}{l|l}
\hline \multicolumn{2}{l}{ Dement Geriatr Cogn Disord 2015;40:276-289 } \\
\hline DOI: $10.1159 / 000437092$ & $\begin{array}{l}\text { C 2 2015 S. Karger AG, Basel } \\
\text { www.karger.com/dem }\end{array}$ \\
\hline
\end{tabular}

Phung et al.: Performance of the 16-Item Informant Questionnaire on Cognitive Decline for the Elderly (IQCODE) in an Arabic-Speaking Older Population

\section{Acknowledgements}

The study was funded by the Fogarty International Center, American National Institutes of Health and National Institute of Aging, grant No. 1R21AG039333-01, under the program 'Brain Disorders in the Developing World: Research across the Lifespan (BRAIN)'. The content is solely the responsibility of the authors and does not represent the official views of the funding agencies. The funding agency had no role in the study design; in the collection, analysis, and interpretation of the data; in the writing of the report, and in the decision to submit the paper for publication.

The study was conducted at the Faculty of Health Sciences, American University of Beirut, Lebanon, in collaboration with the Department of Neurology at the American University of Beirut; the Danish Dementia Research Center, Department of Neurology, Rigshospitalet, University of Copenhagen, Copenhagen, Denmark, and the Department of Health Service and Population Research, Institute of Psychiatry, King's College London, London, UK. The recruitment was a community effort with contribution from institutions, organizations, and individuals. Above all, we would like to express our deep gratitude to the participants in the study. We would also like to thank the Ministry of Social Affairs' Social Center in Bourj Hammoud; the NGO's Restaurant du Coeur, the ladies from the Board of the Child and Mother Welfare Society, and the Ayadina Social Center for the Elderly; the Ain Wazein Hospital Geriatric Clinic; the Neurology Clinic at the American University of Beirut Medical Center; the geriatric clinics at the Al Saydeh Hospital in Antelias, the Child and Mother Welfare Society, and Baytouna Nursing Home; the primary care clinics at the Maronite Nursing Home, Al Saydeh Church in Hamra, and Al Salib Church in Naaba; the social workers Glady Farrah and Mona Saliby; Mrs. Dora Chaaya; Dean Iman Nuwayhid and Prof. Jihad Makhoul from the Faculty of Health Sciences, American University of Beirut; Mrs. Helen Samaha, President of the Lebanese Order of Nursing, and Dr. Raja Sawayaand and Dr. Nazem Bassil, for their assistance with recruitment. We thank Dr. Ibrahim Zeinaty, Dr. Sandrine Salman, Dr. Salem Hammoud, Dr. Wael Radwan, and Dr. Ali Al Ahmar for their work as interviewers in the study. Finally, we express our gratitude to the Alzheimer Association Lebanon for providing the educational materials about dementia for the patients and their caregivers in this study.

\section{Disclosure Statement}

The authors declare that they have no conflicts of interest.

\section{References}

1 Sibai AM, Sen K, Baydoun M, Saxena P: Population ageing in Lebanon: current status, future prospects and implications for policy. Bull World Health Organ 2004;82:219-225.

-2 Prince M, Bryce R, Albanese E, Wimo A, Ribeiro W, Ferri CP: The global prevalence of dementia: a systematic review and metaanalysis. Alzheimers Dement 2013;9:63-75.

-3 Folstein MF, Folstein SE, McHugh PR: 'Mini-mental state'. A practical method for grading the cognitive state of patients for the clinician. J Psychiatr Res 1975;12:189-198.

-4 Nasreddine ZS, Phillips NA, Bédirian V, Charbonneau S, Whitehead V, Collin I, et al: The Montreal Cognitive Assessment, MoCA: a brief screening tool for mild cognitive impairment. J Am Geriatr Soc 2005;53:695-699.

5 Ardila A, Bertolucci PH, Braga LW, Castro-Caldas A, Judd T, Kosmidis MH, Matute E, Nitrini R, Ostrosky-Solis F, Rosselli M: Illiteracy: the neuropsychology of cognition without reading. Arch Clin Neuropsychol 2010;25: 689-712.

6 Jorm AF, Scott R, Jacomb PA: Assessment of cognitive decline in dementia by informant questionnaire. Int J Geriatr Psychiatry 1989;4:35-39.

7 Lin JS, O'Connor E, Rossom RC, Perdue LA, Eckstrom E: Screening for cognitive impairment in older adults: a systematic review for the US Preventive Services Task Force. Ann Intern Med 2013;159:601-612.

-8 Stratford JA, LoGiudice D, Flicker L, Cook R, Waltrowicz W, Ames D: A memory clinic at a geriatric hospital: a report on 577 patients assessed with the CAMDEX over 9 years. Aust NZ J Psychiatry 2003;37:319-326.

9 Flicker L, LoGiudice D, Carlin JB, Ames D: The predictive value of dementia screening instruments in clinical populations. Int J Geriatr Psychiatry 1997;12:203-209.

$\checkmark 10$ Morales JM, Bermejo F, Romero M, Del-Ser T: Screening of dementia in community-dwelling elderly through informant report. Int J Geriatr Psychiatry 1997;12:808-816.

11 de Jonghe JFM: Differentiating between demented and psychiatric patients with the Dutch version of the IQCODE. Int J Geriatr Psychiatry 1997;12:462-465. 
Phung et al.: Performance of the 16-Item Informant Questionnaire on Cognitive Decline for the Elderly (IQCODE) in an Arabic-Speaking Older Population

$>12$ Fuh JL, Teng EL, Lin KN, Larson EB, Wang SJ, Liu CY, Chou P, Kuo BI, Lui HC: The Informant Questionnaire on Cognitive Decline in the Elderly (IQCODE) as a screening tool for dementia for a predominantly illiterate Chinese population. Neurology 1995;45:92-96.

-13 Perroco TR, Bustamante SEZ, Moreno Mdel P, Hototian SR, Lopes MA, Azevedo D, Litvoc J, Filho WJ, Bottino $\mathrm{CM}$ : Performance of the Brazilian long and short IQCODE on the screening of dementia in elderly people with low education. Int Psychogeriatr 2009;21:531-538.

14 Jorm AF: A short form of the Informant Questionnaire on Cognitive Decline in the Elderly (IQCODE): development and cross-validation. Psychol Med 1994;24:145-153.

15 Phung KT, Chaaya M, Waldemar G, Atweh S, Asmar K, Ghusn H, Karam G, Sawaya R, Khoury RM, Zeinaty I, Salman S, Hammoud S, Radwan W, Bassil N, Prince M: Validation of the 10/66 Dementia Research Group diagnostic assessment for dementia in Arabic: a study in Lebanon. J Geriatr Psychiatry Neurol 2014;27:282-290.

$>16$ Chaaya M, Phung KT, El Asmar K, Atweh S, Ghusn H, Khoury RM, Prince M, Nielsen TR, Waldemar G: Validation of the Arabic Rowland Universal Dementia Assessment Scale (A-RUDAS) in elderly with mild and moderate dementia. Aging Ment Health 2015;18:1-8.

$\checkmark 17$ Jorm AF, Broe GA, Creasey H, Sulway MR, Dent O, Fairley MJ, Kos SC, Tennent C: Further data on the validity of the Informant Questionnaire on Cognitive Decline in the Elderly (IQCODE). Int J Geriatr Psychiatry 1996; 11:131-139.

18 Harwood DM, Hope T, Jacoby R: Cognitive impairment in medical inpatients. I. Screening for dementia - is history better than mental state? Age Ageing 1997;26:31-35.

19 Jorm AF: The Informant Questionnaire on Cognitive Decline in the Elderly (IQCODE): a review. Int Psychogeriatr 2004;16:275-293.

20 Barba R, Martínez-Espinosa S, Rodríguez-García E, Pondal M, Vivancos J, Del Ser T: Poststroke dementia: clinical features and risk factors. Stroke 2000;31:1494-1501.

21 Pisani MA, Inouye SK, McNicoll L, Redlich CA: Screening for preexisting cognitive impairment in older intensive care unit patients: use of proxy assessment. J Am Geriatr Soc 2003;51:689-693.

22 World Health Organization: The ICD-10 Classification of Mental and Behavioral Disorders Diagnostic Criteria for Research. Geneva, World Health Organization, 1993.

-23 Albert MS, DeKosky ST, Dickson D, Dubois B, Feldman HH, Fox NC, Gamst A, Holtzman DM, Jagust WJ, Petersen RC, Snyder PJ, Carrillo MC, Thies B, Phelps CH: The diagnosis of mild cognitive impairment due to Alzheimer's disease: recommendations from the National Institute on Aging-Alzheimer's Association workgroups on diagnostic guidelines for Alzheimer's disease. Alzheimers Dement 2011;7:270-279.

24 American Psychiatric Association: Diagnostic and Statistical Manual of Mental Disorders, ed 4, text rev. Washington, American Psychiatric Association, 2000.

25 Morris JC: The Clinical Dementia Rating (CDR): current version and scoring rules. Neurology 1993;43:24122414.

-26 Copeland JR, Dewey ME, Griffiths-Jones HM: A computerized psychiatric diagnostic system and case nomenclature for elderly subjects: GMS and AGECAT. Psychol Med 1986;16:99.

27 Prince M, Acosta D, Chiu H, Copeland J, Dewey M, Scazufca M, Varghese M: Effects of education and culture on the validity of the Geriatric Mental State and its AGECAT algorithm. Br J Psychiatry 2004;185:429-436.

-28 Rahman TT, El Gaafary MM: Montreal Cognitive Assessment Arabic version: reliability and validity prevalence of mild cognitive impairment among elderly attending geriatric clubs in Cairo. Geriatr Gerontol Int 2009;9: $54-61$.

29 Inzelberg R, Schechtman E, Abuful A, Masarwa M, Mazarib A, Strugatsky R, Farrer LA, Friedland RP: Education effects on cognitive function in a healthy aged Arab population. Int Psychogeriatr 2007;19:593-603.

-30 Dunn LB, Lindamer LA, Palmer BW, Schneiderman LJ, Jeste DW: Enhancing comprehension of consent for research in older patients with psychosis: a randomized study of a novel consent procedure. Am J Psychiatry 2001;158:1911-1913.

-31 Wirshing DA, Wirshing WC, Marder SR, Liberman RP, Mintz J: Informed consent: assessment of comprehension. Am J Psychiatry 1998;155:1508-1511.

-32 Mackinnon A, Khalilian A, Jorm AF, Korten AE, Christensen H, Mulligan A: Improving screening accuracy for dementia in a community sample by augmenting cognitive testing with informant report. J Clin Epidemiol 2003;56:358-366.

-33 Ehrensperger MM, Taylor KI, Berres M, Foldi NS, Dellenbach M, Bopp I, Gold G, von Gunten A, Inglin D, Müri R, Rüegger B, Kressig RW, Monsch AU: BrainCheck - a very brief tool to detect incipient cognitive decline: optimized case-finding combining patient- and informant-based data. Alzheimers Res Ther 2014;6:69. 